\title{
Almost Periodic Solutions for Wilson-Cowan Type Model with Time-Varying Delays
}

\author{
Shasha Xie and Zhenkun Huang \\ School of Science, Jimei University, Xiamen 361021, China \\ Correspondence should be addressed to Zhenkun Huang; hzk974226@jmu.edu.cn
}

Received 9 October 2012; Accepted 26 December 2012

Academic Editor: Junli Liu

Copyright ( 2013 S. Xie and Z. Huang. This is an open access article distributed under the Creative Commons Attribution License, which permits unrestricted use, distribution, and reproduction in any medium, provided the original work is properly cited.

Wilson-Cowan model of neuronal population with time-varying delays is considered in this paper. Some sufficient conditions for the existence and delay-based exponential stability of a unique almost periodic solution are established. The approaches are based on constructing Lyapunov functionals and the well-known Banach contraction mapping principle. The results are new, easily checkable, and complement existing periodic ones.

\section{Introduction}

Consider a well-known Wilson-Cowan type model $[1,2]$ with time-varying delays

$$
\begin{aligned}
\frac{d X_{P}(t)}{d t}= & -X_{P}(t)+\left[k_{P}-r_{P} X_{P}(t)\right] \\
& \times G\left[w_{P}^{1} X_{P}\left(t-\tau_{P}(t)\right)\right. \\
& \left.-w_{N}^{1} X_{N}\left(t-\tau_{N}(t)\right)+I_{P}(t)\right], \\
\frac{d X_{N}(t)}{d t}= & -X_{N}(t)+\left[k_{N}-r_{N} X_{N}(t)\right] \\
& \times G\left[w_{P}^{2} X_{P}\left(t-\tau_{P}(t)\right)\right. \\
& \left.-w_{N}^{2} X_{N}\left(t-\tau_{N}(t)\right)+I_{N}(t)\right],
\end{aligned}
$$

where $X_{P}(t), X_{N}(t)$ represent the proportion of excitatory and inhibitory neurons firing per unit time at the instant $t$, respectively. $r_{P}$ and $r_{N}$ are related to the duration of the refractory period, $k_{P}$ and $k_{N}$ are constants. $w_{P}^{1}, w_{N}^{1}, w_{P}^{2}$, and $w_{N}^{2}$ are the strengths of connections between the populations. $I_{P}(t), I_{N}(t)$ are the external inputs to the excitatory and the inhibitory populations. $G(\cdot)$ is the response function of neuronal activity and it is always assumed to be sigmoid type.
$\tau_{P}(t), \tau_{N}(t)$ correspond to the transmission time-varying delays.

It is interesting to revisit Wilson-Cowan system on the following points.

(i) The Wilson-Cowan model has a realistic biological background which describes interactions between excitatory and inhibitory populations of neurons [13]. It has extensive application such as pattern analysis and image processing $[4,5]$.

(ii) There exists rich dynamical behavior in WilsonCowan model. Theoretical results about stable limit cycles, equilibria, chaos, and oscillatory activity have been reported in [2, 3, 6-9]. Recently, Decker and Noonburg [8] reported new results about the existence of three periodic solutions when each neuron was stimulated by periodical inputs. However, under time-varying (periodic or almost periodic) inputs, Wilson-Cowan model can have more complex state space and coexistence of divergent solutions and local stable solutions which could not be easily estimated by its boundary. To see this, we can refer to Figure 1 for the phase portrait of solutions of the following 


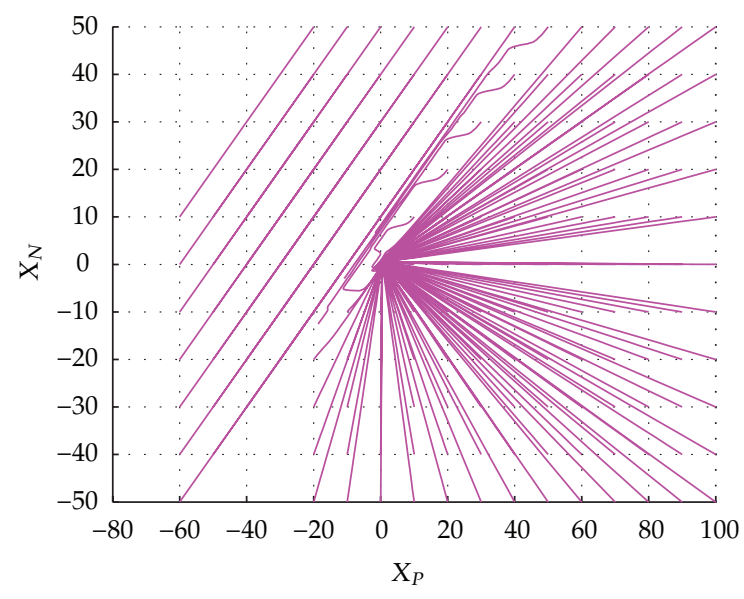

FIGURE 1: Coexistence of divergent and local stable solutions of (2) with almost periodic inputs.

Wilson-Cowan type model with $G(z)=\tanh (z)$ and almost periodic inputs [10-12]:

$$
\begin{aligned}
\frac{d X_{P}(t)}{d t}= & -X_{P}(t)+\left[1-X_{P}(t)\right] \\
& \times G\left[X_{P}(t)-X_{N}(t)+5 \sin \sqrt{2} t\right], \\
\frac{d X_{N}(t)}{d t}= & -X_{N}(t)+\left[1-X_{N}(t)\right] \\
& \times G\left[X_{P}(t)-X_{N}(t)+0.5 \cos t \sin t\right] .
\end{aligned}
$$

(iii) Few works reported almost periodicity of WilsonCowan type model in the literature. Under almost periodic inputs, whether there exists a unique almost periodic solution of (1) which is stable? How to estimate its located boundary? Revealing these results can give a significant insight into the complex dynamical structure of Wilson-Cowan type model.

Throughout this paper, we always assume that $k_{P}, k_{N}, r_{P}$, $r_{N}, w_{P}^{1}, w_{P}^{2}, w_{N}^{1}$, and $w_{N}^{2}$ are positive constants, $\tau_{P}(t), \tau_{N}(t)$, $I_{P}(t)$, and $I_{N}(t)$ are almost periodic functions [12], and set

$$
\begin{array}{ll}
\tau_{P}^{\top}=\sup _{t \in \mathbb{R}} \tau_{P}(t), & \tau_{N}^{\top}=\sup _{t \in \mathbb{R}} \tau_{N}(t), \\
I_{P}^{\top}=\sup _{t \in \mathbb{R}}\left|I_{P}(t)\right|, & I_{N}^{\top}=\sup _{t \in \mathbb{R}}\left|I_{N}(t)\right| .
\end{array}
$$

Moreover, we need some basic assumptions in this paper.

$\left(H_{1}\right) G(0)=0, \sup _{v \in \mathbb{R}}|G(v)| \leqslant B_{s}$ and there exists an $L>$ 0 such that

$$
|G(u)-G(v)| \leqslant L|u-v| \quad \text { for } \forall u, v \in \mathbb{R} .
$$

$\left(H_{2}\right)$ The quadratic equation $(K+R x)(I+W x)=x$ has a positive solution $\delta$, where

$$
\begin{gathered}
W=\max \left\{L\left(w_{P}^{1}+w_{N}^{1}\right), L\left(w_{P}^{2}+w_{N}^{2}\right)\right\}, \\
K=\max \left\{k_{P}, k_{N}\right\}, \quad R=\max \left\{r_{P}, r_{N}\right\}, \\
I=\max \left\{L I_{P}^{\top}, L I_{N}^{\top}\right\} .
\end{gathered}
$$

$\left(H_{3}\right) \tau_{P}(t), \tau_{N}(t)$ are bounded and continuously differentiable with $0 \leq \tau_{P}(t) \leq \tau_{P}^{\top}$,

$$
\begin{array}{cl}
0 \leq \tau_{N}(t) \leq \tau_{N}^{\top}, & 1-\dot{\tau}_{P}(t)>0, \\
1-\dot{\tau}_{N}(t)>0 & \text { for } t \in R .
\end{array}
$$

For for all $u=\left(u_{1}, u_{2}\right) \in \mathbb{R}^{2}$, we define the norm $\|u\|=$ $\max \left\{\left|u_{1}\right|,\left|u_{2}\right|\right\}$. Let $B:=\left\{\psi \mid \psi=\left(\psi_{1}, \psi_{1}\right)\right\}$, where $\psi$ is an almost periodic function on $\mathbb{R}^{2}$. For all $\psi \in B$, if we define induced nodule $\|\psi\|_{B}=\sup _{t \in \mathbb{R}}\|\psi(t)\|$, then $B$ is a Banach space. The initial conditions of system (1) are of the form

$$
X_{P}(s)=\psi_{P}(s), \quad X_{N}(s)=\psi_{N}(s), \quad s \in[-\tau, 0],
$$

where $\tau=\max \left\{\tau_{P}^{\top}, \tau_{N}^{\top}\right\}$ and $\psi=\left(\psi_{P}, \psi_{N}\right) \in B$.

Definition 1 (see [12]). Let $u(t): \mathbb{R} \rightarrow \mathbb{R}^{n}$ be continuous. $u(t)$ is said to be almost periodic on $\mathbb{R}$ if, for any $\varepsilon>0$, it is possible to find a real number $l=l(\varepsilon)>0$, and for any interval with length $l(\varepsilon)$, there exists a number $\delta=\delta(\varepsilon)$ in this interval such that $|u(t+\delta)-u(t)|<\varepsilon$, for all $t \in \mathbb{R}$.

The remaining part of this paper is organized as follows. In Section 2, we will derive sufficient conditions for checking the existence of almost periodic solutions. In Section 3, we present delay-based exponential stability of the unique almost periodic solution of system (1). In Section 4, we will give an example to illustrate our results obtained in the preceding sections. Concluding remarks are given in Section 5.

\section{Existence of Almost Periodic Solutions}

Theorem 2. Suppose that $\left(H_{1}\right)$ and $\left(H_{2}\right)$ hold. If $K W+R\left(B_{s}+\right.$ $\delta W)<1$, then there exists a unique almost periodic solution of system (1) in the region

$$
B^{*}=\left\{\psi \mid \psi \in B,\|\psi\|_{B} \leq \delta\right\}
$$


Proof. For for all $\psi=\left(\psi_{P}, \psi_{N}\right) \in B^{*}$, we consider the almost periodic solution $X^{\psi}(t)=\left(X_{P}^{\psi}(t), X_{N}^{\psi}(t)\right)$ of the following almost periodic differential equations:

$$
\begin{aligned}
\frac{d X_{P}(t)}{d t}= & -X_{P}(t)+\left[k_{P}-r_{P} \psi_{P}(t)\right] \\
& \times G\left[w_{P}^{1} \psi_{P}\left(t-\tau_{P}(t)\right)\right. \\
& \left.-w_{N}^{1} \psi_{N}\left(t-\tau_{N}(t)\right)+I_{P}(t)\right], \\
\frac{d X_{N}(t)}{d t}= & -X_{N}(t)+\left[k_{N}-r_{N} \psi_{N}(t)\right] \\
& \times G\left[w_{P}^{2} \psi_{P}\left(t-\tau_{P}(t)\right)\right. \\
& \left.-w_{N}^{2} \psi_{N}\left(t-\tau_{N}(t)\right)+I_{N}(t)\right] .
\end{aligned}
$$

By almost periodicity of $\tau_{P}(t), \tau_{N}(t), I_{P}(t)$, and $I_{N}(t)$ and Theorem 3.4 in [12] or [10], (9) has a unique almost periodic solution

$$
\begin{gathered}
X_{P}^{\psi}(t) \\
=\int_{-\infty}^{t} \exp (-(t-s))\left[k_{P}-r_{P} \psi_{P}(s)\right] \\
\times G\left[w_{P}^{1} \psi_{P}\left(s-\tau_{P}(s)\right)\right. \\
\left.-w_{N}^{1} \psi_{N}\left(s-\tau_{N}(s)\right)+I_{P}(s)\right] d s, \\
X_{N}^{\psi}(t)=\int_{-\infty}^{t} \exp (-(t-s))\left[k_{N}-r_{N} \psi_{N}(s)\right] \\
\times G\left[w_{P}^{2} \psi_{P}\left(s-\tau_{P}(s)\right)\right. \\
\left.-w_{N}^{2} \psi_{N}\left(s-\tau_{N}(s)\right)+I_{N}(s)\right] d s .
\end{gathered}
$$

Define a mapping $F: B^{*} \rightarrow B$ by setting $F(\psi)(t)=$ $\left(F_{P}(\psi)(t), F_{N}(\psi)(t)\right)=X^{\psi}(t)$, for all $\psi \in B^{*}$. Now, we prove that $F$ is a self-mapping from $B^{*}$ to $B^{*}$. From $(10)$ and $\left(H_{1}\right)$, we obtain

$$
\begin{aligned}
& \left|F_{P}(\psi)(t)\right| \\
& \leq \sup _{t \in \mathbb{R}} \mid \int_{-\infty}^{t} \exp (-(t-s))\left[k_{P}-r_{P} \psi_{P}(s)\right] \\
& \quad \times G\left[w_{P}^{1} \psi_{P}\left(s-\tau_{P}(s)\right)\right. \\
& \left.\quad-w_{N}^{1} \psi_{N}\left(s-\tau_{N}(s)\right)+I_{P}(s)\right] d s \mid
\end{aligned}
$$

$$
\begin{aligned}
& \leq \sup _{t \in \mathbb{R}}\left[k_{P}+r_{P}\left|\psi_{P}(t)\right|\right] \\
& \quad \times G \mid\left[w_{P}^{1} \psi_{P}\left(t-\tau_{P}(t)\right)\right. \\
& \left.\quad-w_{N}^{1} \psi_{N}\left(t-\tau_{N}(t)\right)+I_{P}(t)\right] \mid \\
& \leq\left(k_{P}+r_{P}\|\psi\|_{B}\right) \\
& \quad \times L\left(w_{P}^{1}\|\psi\|_{B}+w_{N}^{1}\|\psi\|_{B}+I_{P}^{\top}\right) \\
& \leq\left(k_{P}+r_{P}\|\psi\|_{B}\right) \\
& \quad \times\left(L\left(w_{P}^{1}+w_{N}^{1}\right)\|\psi\|_{B}+L I_{P}^{\top}\right) .
\end{aligned}
$$

By similar estimation, we can get

$$
\begin{aligned}
\left|F_{N}(\psi)\right| \leq & \left(k_{N}+r_{N}\|\psi\|_{B}\right) \\
& \times\left(L\left(w_{P}^{2}+w_{N}^{2}\right)\|\psi\|_{B}+L I_{N}^{\top}\right) .
\end{aligned}
$$

Therefore, by the above estimations and $\left(\mathrm{H}_{2}\right)$, we get

$$
\begin{aligned}
\|F(\psi)\|_{B} & =\sup _{t \in \mathbb{R}}\left\{\left|F_{P}(\psi)\right|,\left|F_{N}(\psi)\right|\right\} \\
& \leq\left(K+R\|\psi\|_{B}\right)\left(W\|\psi\|_{B}+I\right) \\
& \leq(K+R \delta)(W \delta+I)=\delta,
\end{aligned}
$$

which implies that $F(\psi) \in B^{*}$. So, the mapping $F$ is selfmapping from $B^{*}$ to $B^{*}$. Next, we prove that $F$ is a contraction mapping in the region $B^{*}$. For all $\psi, \phi \in B^{*}$, by (10), we have

$$
\begin{aligned}
& \left|F_{P}(\psi)(t)-F_{P}(\phi)(t)\right| \\
& \leq \sup _{t \in \mathbb{R}} \mid \int_{-\infty}^{t} \exp (-(t-s))\left[k_{P}-r_{P} \psi_{P}(s)\right] \\
& \times G\left[w_{P}^{1} \psi_{P}\left(s-\tau_{P}(s)\right)\right. \\
& \left.-w_{N}^{1} \psi_{N}\left(s-\tau_{N}(s)\right)+I_{P}(s)\right] d s \\
& -\int_{-\infty}^{t} \exp (-(t-s))\left[k_{P}-r_{P} \phi_{P}(s)\right] \\
& \times G\left[w_{P}^{1} \phi_{P}\left(s-\tau_{P}(s)\right)\right. \\
& \left.\quad-w_{N}^{1} \phi_{N}\left(s-\tau_{N}(s)\right)+I_{P}(s)\right] d s \mid,
\end{aligned}
$$


which leads to

$$
\begin{aligned}
& \left|F_{P}(\psi)(t)-F_{P}(\phi)(t)\right| \\
& \leq \sup _{t \in \mathbb{R}} \int_{-\infty}^{t} \exp (-(t-s)) \\
& \times\left[k_{P} L \mid w_{P}^{1} \psi_{P}\left(s-\tau_{P}(s)\right)-w_{N}^{1} \psi_{N}\left(s-\tau_{N}(s)\right)\right. \\
& -w_{P}^{1} \phi_{P}\left(s-\tau_{P}(s)\right)+w_{N}^{1} \phi_{N}\left(s-\tau_{N}(s)\right) \\
& +r_{P} \mid \psi_{P}(s) G\left[w_{P}^{1} \psi_{P}\left(s-\tau_{P}(s)\right)\right. \\
& -w_{N}^{1} \psi_{N}\left(s-\tau_{N}(s)\right) \\
& \left.+I_{P}(s)\right] \\
& -\phi_{P}(s) G\left[w_{P}^{1} \phi_{P}\left(s-\tau_{P}(s)\right)\right. \\
& -w_{N}^{1} \phi_{N}\left(s-\tau_{N}(s)\right) \\
& \left.\left.+I_{P}(s)\right] \mid\right] d s \\
& \leq k_{P} L\left(w_{P}^{1} \sup _{t \in \mathbb{R}}\left|\psi_{P}(t)-\phi_{P}(t)\right|+w_{N}^{1} \sup _{t \in \mathbb{R}}\left|\psi_{N}(t)-\phi_{N}(t)\right|\right) \\
& +\sup _{t \in \mathbb{R}} \int_{-\infty}^{t} \exp (-(t-s)) r_{P} \\
& \times\left[\mid \psi_{P}(s) G\left(w_{P}^{1} \psi_{P}\left(s-\tau_{P}(s)\right)\right.\right. \\
& \left.-w_{N}^{1} \psi_{N}\left(s-\tau_{N}(s)\right)+I_{P}(s)\right) \\
& -\psi_{P}(s) G\left(w_{P}^{1} \phi_{P}\left(s-\tau_{P}(s)\right)\right. \\
& -w_{N}^{1} \phi_{N}\left(s-\tau_{N}(s)\right) \\
& \left.+I_{P}(s)\right) \\
& +\mid \psi_{P}(s) G\left(w_{P}^{1} \phi_{P}\left(s-\tau_{P}(s)\right)\right. \\
& \left.-w_{N}^{1} \phi_{N}\left(s-\tau_{N}(s)\right)+I_{P}(s)\right) \\
& -\phi_{P}(s) G\left(w_{P}^{1} \phi_{P}\left(s-\tau_{P}(s)\right)\right. \\
& \left.\left.-w_{N}^{1} \phi_{N}(s)+I_{P}(s)\right) \mid\right] \mid d s \\
& \leq k_{P} L\left(w_{P}^{1}+w_{N}^{1}\right)\|\psi-\phi\|_{B} \\
& +r_{P}\left(B_{s}\|\psi-\phi\|_{B}+\delta L\left(w_{P}^{1}+w_{N}^{1}\right)\|\psi-\phi\|_{B}\right) .
\end{aligned}
$$

By similar argument, we can get

$$
\begin{aligned}
& \left|F_{N}(\psi)(t)-F_{N}(\psi)(t)\right| \\
& \quad \leq k_{N} L\left(w_{P}^{2}+w_{N}^{2}\right)\|\psi-\phi\|_{B} \\
& \quad+r_{N}\left(B_{s}\|\psi-\phi\|_{B}+\delta L\left(w_{P}^{2}+w_{N}^{2}\right)\|\psi-\phi\|_{B}\right) .
\end{aligned}
$$

From (15) and (16), we have

$$
\begin{aligned}
\| F & (\psi)-F(\psi) \|_{B} \\
= & \sup _{t \in \mathbb{R}}\left\{\left|F_{P}(\psi)-F_{P}(\psi)\right|,\left|F_{N}(\psi)-F_{N}(\psi)\right|\right\} \\
\leq & K W\|\psi-\phi\| \\
& +R\left(B_{s}\|\psi-\phi\|+\delta W\|\psi-\phi\|\right) \\
= & \left(K W+R B_{s}+R \delta W\right)\|\psi-\phi\| .
\end{aligned}
$$

Since $K W+R B_{s}+R \delta W \in(0,1)$, it is clear that the mapping $F$ is a contraction. Therefore the mapping $F$ possesses a unique fixed point $X^{*} \in B^{*}$ such that $F X^{*}=X^{*}$. By (9), $X^{*}$ is an almost periodic solution of system (1) in $B^{*}$. The proof is complete.

Remark 3. Obviously, quadratic curve $\mathscr{C}(v):=R W v^{2}+(K W+$ $R I-1) v+K I$ satisfies with $\mathscr{C}(0) \geq 0$. So, $\Delta:=(K W+R I-$ $1)^{2}-4 R W K I>0$ and $K W+R I<1$ guarantees the existence of $\delta$ in $\left(\mathrm{H}_{2}\right)$ and $\delta$ lies in the following interval:

$$
\left[\frac{1-K W-R I-\sqrt{\Delta}}{2 R W}, \frac{1-K W-R I+\sqrt{\Delta}}{2 R W}\right] .
$$

By Theorem 2, we know that the unique almost periodic solution depends on $K W+R\left(B_{s}+\delta W\right)<1$. Choosing $\delta=(1-(K W+R I)) / 2 R W$, we get a simple assumption as follows:

$$
\left(\widehat{H}_{2}\right) K W+R I<1-2 \sqrt{K W R I}, R B_{s}<(1-(K W-R I)) / 2,
$$

and hence it leads to a parameter-based result.

Corollary 4. Suppose that $\left(H_{1}\right)$ and $\left(\widehat{H}_{2}\right)$ hold. Then there exists a unique almost periodic solution of system (1) in the region $B^{*}=\left\{\psi \mid \psi \in B,\|\psi\|_{B} \leq(1-(K W+R I)) / 2 R W\right\}$.

\section{Delay-Based Stability of the Almost Periodic Solution}

In this section, we establish locally exponential stability of the unique almost periodic solution of system (1) in the region $B^{*}$, which is delay dependent.

Theorem 5. Suppose that $\left(H_{1}\right)-\left(H_{3}\right)$ hold. If $K W+R\left(B_{s}+\right.$ $\delta W)<1$ and there exist constants $\ell_{1}>0, \ell_{2}>0$ such that

$$
\begin{aligned}
& \left(1-r_{P} B_{s}\right) \ell_{1}>\sup _{t \in \mathbb{R}} \frac{C_{P}}{1-\dot{\tau}_{P}\left(v_{P}^{-1}(t)\right)}, \\
& \left(1-r_{N} B_{s}\right) \ell_{2}>\sup _{t \in \mathbb{R}} \frac{C_{N}}{1-\dot{\tau}_{N}\left(v_{N}^{-1}(t)\right)},
\end{aligned}
$$

where $C_{P}:=L\left[\left(k_{P}+r_{P} \delta\right) \ell_{1} w_{P}^{1}+\left(k_{N}+r_{N} \delta\right) \ell_{2} w_{P}^{2}\right]$ and $C_{N}:=$ $L\left[\left(k_{P}+r_{P} \delta\right) \ell_{1} w_{N}^{1}+\left(k_{N}+r_{N} \delta\right) \ell_{2} w_{N}^{2}\right], v_{P}^{-1}(t)$ and $v_{N}^{-1}(t)$ are the inverse functions of $v_{P}(t)=t-\tau_{P}(t)$ and $v_{N}(t)=t-\tau_{N}(t)$, then system (1) has exactly one almost periodic solution $X^{*}(t)$ in the region $B^{*}$ which is locally exponentially stable. 
Proof. From Theorem 2, system (1) has a unique almost periodic solution $X^{*}(t) \in B^{*}$. Let $X(t)=\left(X_{P}(t), X_{N}(t)\right)$ be an arbitrary solution of system (1) with initial value $\psi=$ $\left(\psi_{P}, \psi_{N}\right) \in B^{*}$. Set $X(t)=X_{P}(t)-X_{P}^{*}(t), Y(t)=X_{N}(t)-$ $X_{N}^{*}(t)$. By system (1), we get

$$
\begin{aligned}
\frac{d X(t)}{d t}= & -X(t) \\
& +\left[k_{P}-r_{P}\left(X(t)+X_{P}^{*}(t)\right)\right] \\
& \times G\left[w_{P}^{1}\left(X\left(t-\tau_{P}(t)\right)+X_{P}^{*}\left(t-\tau_{P}(t)\right)\right)\right. \\
& \left.\quad-w_{N}^{1}\left(Y\left(t-\tau_{N}(t)\right)+X_{N}^{*}\left(t-\tau_{N}(t)\right)\right) I_{P}(t)\right] \\
- & {\left[k_{P}-r_{P} X_{P}^{*}(t)\right] } \\
& \times G\left[w_{P}^{1} X_{P}^{*}\left(t-\tau_{P}(t)\right)\right. \\
& \left.\quad-w_{N}^{1} X_{N}^{*}\left(t-\tau_{N}(t)\right)+I_{P}(t)\right],
\end{aligned}
$$

$\frac{d Y(t)}{d t}=-Y(t)$

$$
\begin{aligned}
& +\left[k_{N}-r_{N}\left(Y(t)+X_{N}^{*}(t)\right)\right] \\
& \times G\left[w_{P}^{2}\left(X\left(t-\tau_{P}(t)\right)+X_{P}^{*}\left(t-\tau_{P}(t)\right)\right)\right. \\
& \left.\quad-w_{N}^{2}\left(Y\left(t-\tau_{N}(t)\right)+X_{N}^{*}\left(t-\tau_{N}(t)\right)\right)+I_{N}(t)\right] \\
& -\left[k_{N}-r_{N} X_{N}^{*}(t)\right] \\
& \times G\left[w_{P}^{2} X_{P}^{*}\left(t-\tau_{P}(t)\right)\right. \\
& \left.\quad-w_{N}^{2} X_{N}^{*}\left(t-\tau_{N}(t)\right)+I_{N}(t)\right] .
\end{aligned}
$$

Construct the auxiliary functions $F_{P}(u), F_{N}(u)$ defined on $[0,+\infty)$ as follows:

$$
\begin{aligned}
& F_{P}(u):=\left(u-1+r_{P} B_{s}\right) \ell_{1}+\sup _{t \in \mathbb{R}} \frac{C_{P} e^{u \tau_{P}^{\top}}}{1-\dot{\tau}_{P}\left(v_{P}^{-1}(t)\right)}, \\
& F_{N}(u):=\left(u-1+r_{N} B_{s}\right) \ell_{2}+\sup _{t \in \mathbb{R}} \frac{C_{N} e^{u \tau_{N}^{\top}}}{1-\dot{\tau}_{N}\left(v_{N}^{-1}(t)\right)} .
\end{aligned}
$$

One can easily show that $F_{P}(u), F_{N}(u)$ are well defined and continuous. Assumption (19) implies that $F_{P}(0)<0$, $F_{P}(u) \rightarrow+\infty$ as $u \rightarrow+\infty$ and $F_{N}(0)<0, F_{N}(u) \rightarrow+\infty$ as $u \rightarrow+\infty$. It follows that there exists a common $\lambda>0$ such that $F_{P}(\lambda)<0$ and $F_{N}(\lambda)<0$.

Consider the Lyapunov functional

$$
\begin{aligned}
V(t)= & {\left[\ell_{1}|X(t)|+\ell_{2}|Y(t)|\right] e^{\lambda t} } \\
& +\int_{t-\tau_{P}(t)}^{t} \frac{C_{P}}{1-\dot{\tau}_{P}\left(v_{P}^{-1}(s)\right)}|X(s)| e^{\lambda\left(s+\tau_{P}^{\top}\right)} d s \\
& +\int_{t-\tau_{N}(t)}^{t} \frac{C_{N}}{1-\dot{\tau}_{N}\left(v_{N}^{-1}(s)\right)}|Y(s)| e^{\lambda\left(s+\tau_{N}^{\top}\right)} d s .
\end{aligned}
$$

Calculating the upper right derivative of $V(t)$ along system (1), one has

$D^{+} V(t)$

$$
\begin{gathered}
\leq \lambda\left[\ell_{1}|X(t)|+\ell_{2}|Y(t)|\right] e^{\lambda t} \\
+e^{\lambda t} \ell_{1}[-|X(t)| \\
+\mid\left[k_{P}-r_{P}\left(X(t)+X_{P}^{*}(t)\right)\right] \\
\times G\left[w_{P}^{1}\left(X\left(t-\tau_{P}(t)\right)+X_{P}^{*}\left(t-\tau_{P}(t)\right)\right)\right. \\
-w_{N}^{1}\left(Y\left(t-\tau_{N}(t)\right)+X_{N}^{*}\left(t-\tau_{N}(t)\right)\right) \\
\left.+I_{P}(t)\right] \\
-\left[k_{P}-r_{P} X_{P}^{*}(t)\right] \\
\times G\left[w_{P}^{1} X_{P}^{*}\left(t-\tau_{P}(t)\right)\right. \\
\left.\left.-w_{N}^{1} X_{N}^{*}\left(t-\tau_{N}(t)\right)+I_{P}(t)\right] \mid\right] \\
+e^{\lambda t} \ell_{2}[-|Y(t)| \\
+\mid\left[k_{N}-r_{N}\left(Y(t)+X_{N}^{*}(t)\right)\right] \\
\times G\left[w_{P}^{2}\left(X\left(t-\tau_{P}(t)\right)+X_{P}^{*}\left(t-\tau_{P}(t)\right)\right)\right. \\
-w_{N}^{2}\left(Y\left(t-\tau_{N}(t)\right)+X_{N}^{*}\left(t-\tau_{N}(t)\right)\right) \\
\left.+I_{N}(t)\right] \\
-\left[k_{N}-r_{N} X_{N}^{*}(t)\right] \\
\times G\left[w_{P}^{2} X_{P}^{*}\left(t-\tau_{P}(t)\right)\right. \\
\left.\left.-w_{N}^{2} X_{N}^{*}\left(t-\tau_{N}(t)\right)+I_{N}(t)\right] \mid\right]
\end{gathered}
$$

$+\frac{C_{P}}{1-\dot{\tau}_{P}\left(v_{P}^{-1}(t)\right)}|X(t)| e^{\lambda\left(t+\tau_{P}^{\top}\right)}$

$-C_{P}\left|X\left(t-\tau_{P}(t)\right)\right| \exp \left[\lambda\left(t-\tau_{P}(t)+\tau_{P}^{\top}\right)\right]$

$+\frac{C_{N}}{1-\dot{\tau}_{N}\left(v_{N}^{-1}(t)\right)}|Y(t)| e^{\lambda\left(t+\tau_{N}^{\top}\right)}$

$-C_{N}\left|Y\left(t-\tau_{N}(t)\right)\right| \exp \left[\lambda\left(t-\tau_{N}(t)+\tau_{N}^{\top}\right)\right]$,

which leads to

$$
\begin{aligned}
& D^{+} V(t) \\
& \leq\left[(\lambda-1) \ell_{1} e^{\lambda t}|X(t)|+(\lambda-1) \ell_{2} e^{\lambda t}|Y(t)|\right] \\
& +e^{\lambda t} \ell_{1}\left[k _ { P } L \left(w_{P}^{1}\left|X\left(t-\tau_{P}(t)\right)\right|\right.\right. \\
& \left.+w_{N}^{1}\left|Y\left(t-\tau_{N}(t)\right)\right|\right)+r_{P}|X(t)| B_{s} \\
& +r_{P} \delta L\left(w_{P}^{1}\left|X\left(t-\tau_{P}(t)\right)\right|\right. \\
& \left.\left.+w_{N}^{1}\left|Y\left(t-\tau_{N}(t)\right)\right|\right)\right]
\end{aligned}
$$




$$
\begin{aligned}
& +e^{\lambda t} l_{2}\left[k _ { N } L \left(w_{P}^{2}\left|X\left(t-\tau_{P}(t)\right)\right|\right.\right. \\
& \left.+w_{N}^{2}\left|Y\left(t-\tau_{N}(t)\right)\right|\right) \\
& +r_{N}|Y(t)| B_{s}+r_{N} \delta L\left(w_{P}^{2}\left|X\left(t-\tau_{P}(t)\right)\right|\right. \\
& \left.\left.+w_{N}^{2}\left|Y\left(t-\tau_{N}(t)\right)\right|\right)\right] \\
& +\frac{C_{P}}{1-\dot{\tau}_{P}\left(v_{P}^{-1}(t)\right)}|X(t)| e^{\lambda\left(t+\tau_{P}^{\top}\right)} \\
& -C_{P}\left|X\left(t-\tau_{P}(t)\right)\right| e^{\lambda t} \\
& +\frac{C_{N}}{1-\dot{\tau}_{N}\left(v_{N}^{-1}(t)\right)}|Y(t)| e^{\lambda\left(t+\tau_{N}^{\top}\right)} \\
& -C_{N}\left|Y\left(t-\tau_{N}(t)\right)\right| e^{\lambda t} \\
& \leq\left[\left(\lambda-1+r_{P} B_{s}\right) \ell_{1}+\sup _{t \in \mathbb{R}} \frac{C_{P} e^{\lambda \tau_{P}^{\top}}}{1-\dot{\tau}_{P}\left(v_{P}^{-1}(t)\right)}\right] \\
& \times e^{\lambda t}|X(t)| \\
& +\left[\left(\lambda-1+r_{N} B_{s}\right) \ell_{2}+\sup _{t \in \mathbb{R}} \frac{C_{N} e^{\lambda \tau_{N}^{\top}}}{1-\dot{\tau}_{N}\left(v_{N}^{-1}(t)\right)}\right] \\
& \leq-c_{1}[|X(t)|+|Y(t)|] e^{\lambda t}, \\
& +[Y(t) \mid
\end{aligned}
$$

where $c_{1}:=-(1 / 2) \max \left\{F_{P}(\lambda), F_{N}(\lambda)\right\}>0$. We have from the above that $V(t) \leq V\left(t_{0}\right)$ and

$$
\min \left\{\ell_{1}, \ell_{2}\right\} e^{\lambda t}(|X(t)|+|Y(t)|) \leq V(t) \leq V(0), \quad t \geq 0 .
$$

Note that

$$
\begin{aligned}
V(0)= & {\left[\left(\ell_{1}+\ell_{2}\right)+\tau_{P}^{\top} \sup _{s \in\left[-\tau_{P}^{\top}, 0\right]} \frac{C_{P}}{1-\dot{\tau}_{P}\left(v_{P}^{-1}(s)\right)} e^{\lambda \tau_{P}^{\top}}\right.} \\
& \left.+\tau_{N}^{\top} \sup _{s \in\left[-\tau_{N}^{\top}, 0\right]} \frac{C_{N}}{1-\dot{\tau}_{N}\left(v_{N}^{-1}(s)\right)} e^{\lambda \tau_{N}^{\top}}\right]\left\|\psi-\psi^{*}\right\|,
\end{aligned}
$$

where $\psi^{*}(s)=X^{*}(s), s \in[-\tau, 0]$. Then there exists a positive constant $M>1$ such that

$$
\begin{aligned}
& \left|X_{P}(t)-X_{P}^{*}(t)\right| \leq M\left\|\psi-\psi^{*}\right\| e^{-\lambda t}, \\
& \left|X_{N}(t)-X_{N}^{*}(t)\right| \leq M\left\|\psi-\psi^{*}\right\| e^{-\lambda t} .
\end{aligned}
$$

The proof is complete.

Set $\delta=(1-(K W+R I)) / 2 R W$. It follows from Corollary 4 and Theorem 5 the following.
Corollary 6. Suppose that $\left(H_{1}\right),\left(\widehat{H}_{2}\right)$, and $\left(H_{3}\right)$ hold. If there exist constants $\ell_{1}>0, \ell_{2}>0$ such that

$$
\begin{gathered}
\left(1-r_{P} B_{s}\right) \ell_{1}>\sup _{t \in \mathbb{R}} \frac{L\left(\alpha_{P} \ell_{1} w_{P}^{1}+\alpha_{N} \ell_{2} w_{P}^{2}\right)}{1-\dot{\tau}_{P}\left(v_{P}^{-1}(t)\right)}, \\
\left(1-r_{N} B_{s}\right) \ell_{2}>\sup _{t \in \mathbb{R}} \frac{L\left(\alpha_{P} \ell_{1} w_{N}^{1}+\alpha_{N} \ell_{2} w_{N}^{2}\right)}{1-\dot{\tau}_{N}\left(v_{N}^{-1}(t)\right)},
\end{gathered}
$$

where

$$
\begin{gathered}
\alpha_{P}:=k_{P}+r_{P} \frac{1-(K W+R I)}{2 R W}, \\
\alpha_{N}:=k_{N}+r_{N} \frac{1-(K W+R I)}{2 R W},
\end{gathered}
$$

$v_{P}^{-1}(t)$ and $v_{N}^{-1}(t)$ are defined as Theorem 5 , then system (1) has exactly one almost periodic solution $X^{*}(t)$ in the region

$$
B^{*}=\left\{\psi \mid \psi \in B,\|\psi\|_{B} \leq \frac{1-(K W+R I)}{2 R W}\right\},
$$

which is locally exponentially stable.

\section{An Example}

In this section, we give an example to demonstrate the results obtained in previous sections. Consider a Wilson-Cowan type model with time-varying delays as follows:

$$
\begin{aligned}
\frac{d X_{P}(t)}{d t}= & -X_{P}(t)+\left[k_{P}-r_{P} X_{P}(t)\right] \\
& \times G\left[w_{P}^{1} X_{P}\left(t-\tau_{P}(t)\right)\right. \\
& \left.-w_{N}^{1} X_{N}\left(t-\tau_{N}(t)\right)+I_{P}(t)\right], \\
\frac{d X_{N}(t)}{d t}= & -X_{N}(t)+\left[k_{N}-r_{N} X_{N}(t)\right] \\
& \times G\left[w_{P}^{2} X_{P}\left(t-\tau_{P}(t)\right)\right. \\
& \left.-w_{N}^{2} X_{N}\left(t-\tau_{N}(t)\right)+I_{N}(t)\right],
\end{aligned}
$$

where $k_{P}=k_{N}=1, r_{P}=r_{N}=0.01, w_{P}^{1}=w_{P}^{2}=w_{N}^{1}=$ $w_{N}^{2}=0.1, \tau_{P}(t)=\tau_{N}(t)=0.1 t+10, I_{P}(t)=7 \sin \sqrt{7} t, G(v)=$ $\tanh (v)$, and $I_{N}(t)=7 \cos \sqrt{2} t$. It is easy to calculate that

$$
\begin{gathered}
K=1, \quad W=0.2, \quad R=0.01, \quad I=7, \\
v_{P}^{-1}(t)=v_{N}^{-1}(t)=\frac{(t+10)}{0.9}, \\
L=B_{s}=1, \quad \delta=182.5 .
\end{gathered}
$$

It is easy to check that $\left(H_{1}\right)$ and $\left(\widehat{H}_{2}\right)$ hold. By Corollary $4,(31)$ has a unique almost periodic solution $X^{*}(t)$ in region $B^{*}=$ $\left\{\psi \mid \psi \in B,\|\psi\|_{B} \leq 182.5\right\}$. Setting $\ell_{1}=\ell_{2}=0.5$, we can check that $\left(H_{3}\right)$ and $(28)$ hold and hence $X^{*}(t)$ is exponentially stable in $B^{*}$. Figure 2 shows the transient behavior of the unique almost periodic solution $\left(X_{P}(t), X_{N}(t)\right)$ in $B^{*}$. Phase portrait of attractivity of $X_{P}$ and $X_{N}$ is illustrated in Figure 3 . 


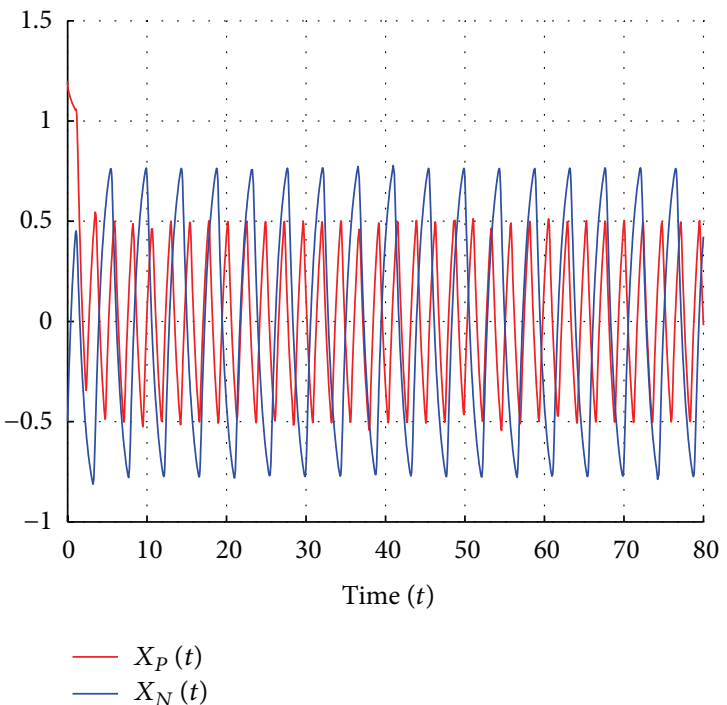

FIgURE 2: Transient behavior of the almost periodic solution of (31) located in $B^{*}$.

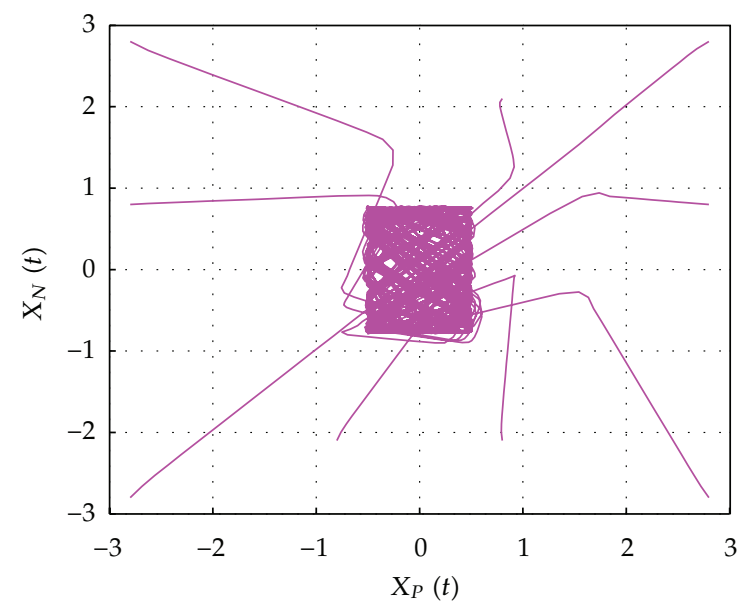

FIgURE 3: Phase portrait of attractivity of the unique almost periodic solution of (31) located in $B^{*}$.

\section{Concluding Remarks}

In this paper, we investigate Wilson-Cowan type model and obtain the existence of a unique almost periodic solution and its delay-based local stability in a convex subset. Our results are new and can reduce to periodic case, hence, complement existing periodic ones $[7,8]$. We point out that there will exist multiple periodic (almost periodic) solution for system (1) under suitable parameter configuration. However, it is difficult to analyze its multistability of almost periodic solution by the existing method $[10,11,13,14]$. We leave it for interested readers.

\section{Acknowledgments}

This research is supported by the National Natural Science Foundation of China under Grants (11101187, 61273021),
NCETFJ (JA11144), the Excellent Youth Foundation of Fujian Province (2012J06001), the Foundation of Fujian High Education (JA10184, JA11154), and the Foundation for Young Professors of Jimei University, China.

\section{References}

[1] H. R. Wilson and J. D. Cowan, "Excitatory and inhibitory interactions in localized populations of model neurons," Biophysical Journal, vol. 12, no. 1, pp. 1-24, 1972.

[2] H. R. Wilson and J. D. Cowan, "A mathematical theory of the functional dynamics of cortical and thalamic nervous tissue," Kybernetik, vol. 13, no. 2, pp. 55-80, 1973.

[3] C. van Vreeswijk and H. Sompolinsky, "Chaos in neuronal networks with balanced excitatory and inhibitory activity," Science, vol. 274, no. 5293, pp. 1724-1726, 1996.

[4] S. E. Folias and G. B. Ermentrout, "New patterns of activity in a pair of interacting excitatory-inhibitory neural fields," Physical Review Letters, vol. 107, no. 22, Article ID 228103, 2011.

[5] K. Mantere, J. Parkkinen, T. Jaaskelainen, and M. M. Gupta, "Wilson-Cowan neural-network model in image processing," Journal of Mathematical Imaging and Vision, vol. 2, no. 2-3, pp. 251-259, 1992.

[6] L. H. A. Monteiro, M. A. Bussab, and J. G. C. Berlinck, "Analytical results on a Wilson-Cowan neuronal network modified model," Journal of Theoretical Biology, vol. 219, no. 1, pp. 83-91, 2002.

[7] B. Pollina, D. Benardete, and V. W. Noonburg, "A periodically forced Wilson-Cowan system," SIAM Journal on Applied Mathematics, vol. 63, no. 5, pp. 1585-1603, 2003.

[8] R. Decker and V. W. Noonburg, "A periodically forced WilsonCowan system with multiple attractors," SIAM Journal on Mathematical Analysis, vol. 44, no. 2, pp. 887-905, 2012.

[9] P. L. William, "Equilibrium and stability of wilson and cowan's time coarse graining model," in Proceedings of the 20th International Symposium on Mathematical Theory of Networks and Systems (MTNS '12), Melbourne, Australia, July 2012.

[10] Z. Huang, S. Mohamad, X. Wang, and C. Feng, "Convergence analysis of general neural networks under almost periodic stimuli," International Journal of Circuit Theory and Applications, vol. 37, no. 6, pp. 723-750, 2009.

[11] Y. Liu and Z. You, "Multi-stability and almost periodic solutions of a class of recurrent neural networks," Chaos, Solitons and Fractals, vol. 33, no. 2, pp. 554-563, 2007.

[12] C. Y. He, Almost Periodic Differential Equation, Higher Education, Beijing, China, 1992.

[13] H. Zhenkun, F. Chunhua, and S. Mohamad, "Multistability analysis for a general class of delayed Cohen-Grossberg neural networks," Information Sciences, vol. 187, pp. 233-244, 2012.

[14] H. Zhenkun and Y. N. Raffoul, "Biperiodicity in neutral-type delayed difference neural networks," Advances in Difference Equations, vol. 2012, article 5, 2012. 


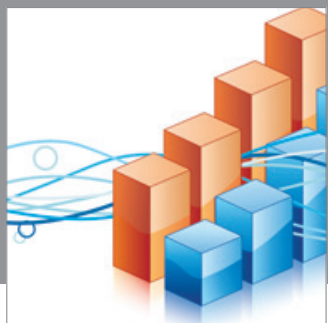

Advances in

Operations Research

mansans

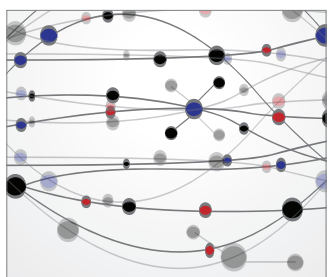

The Scientific World Journal
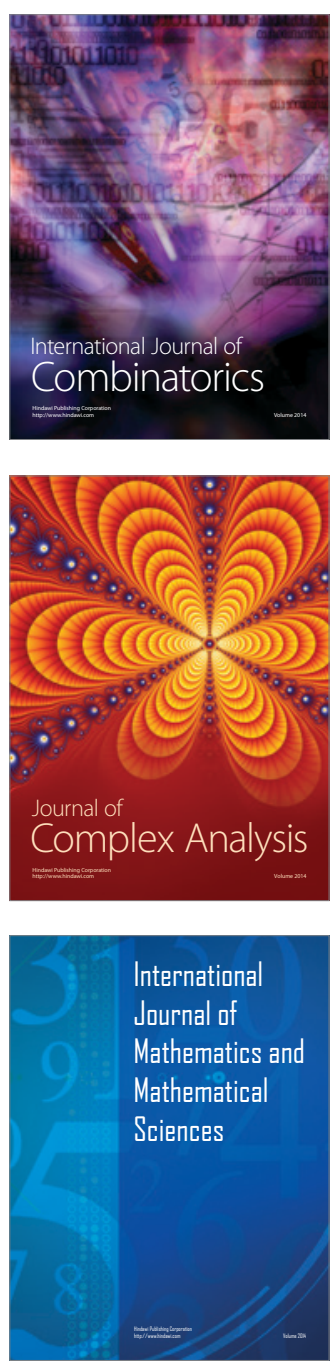
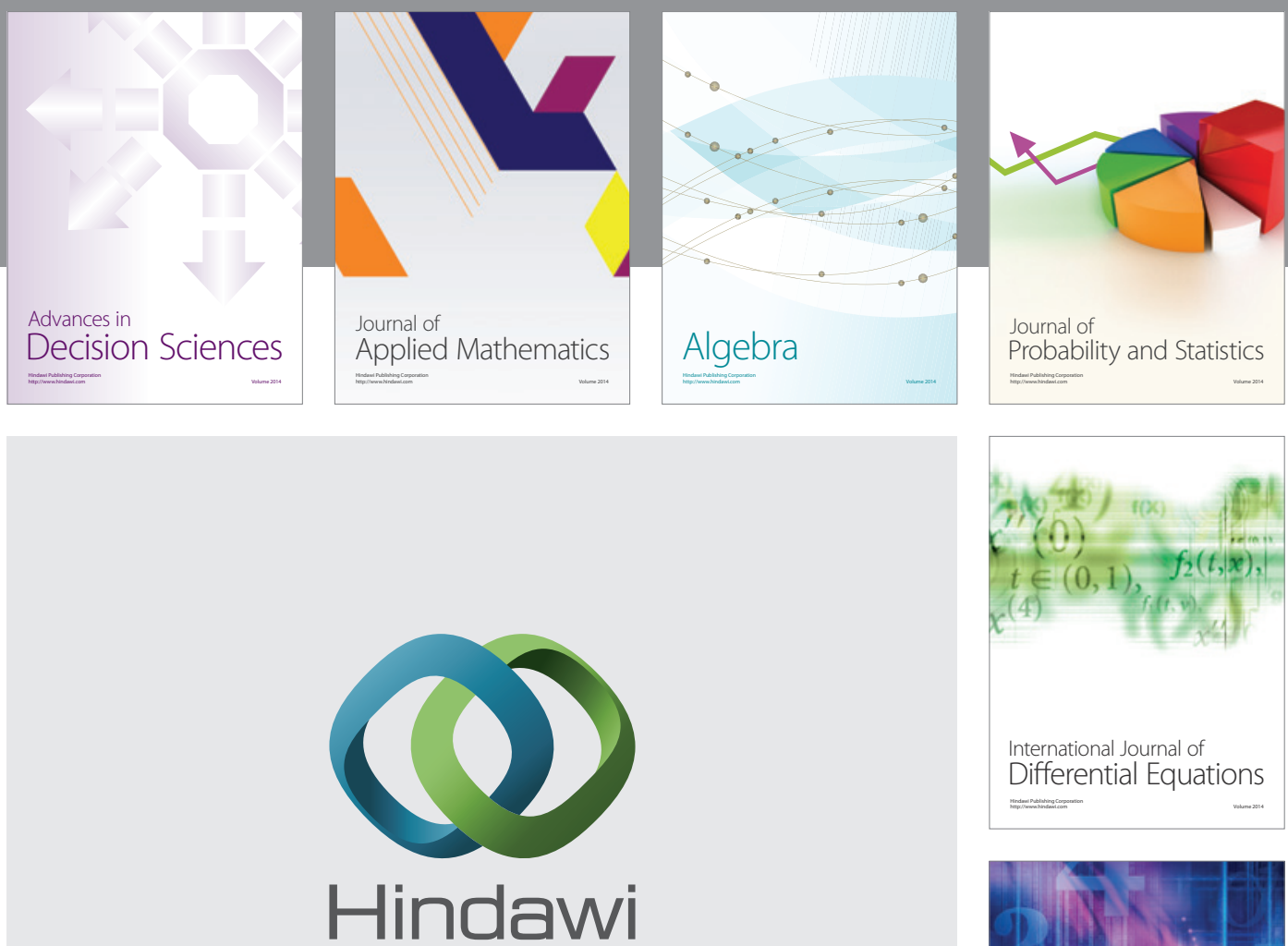

Submit your manuscripts at http://www.hindawi.com
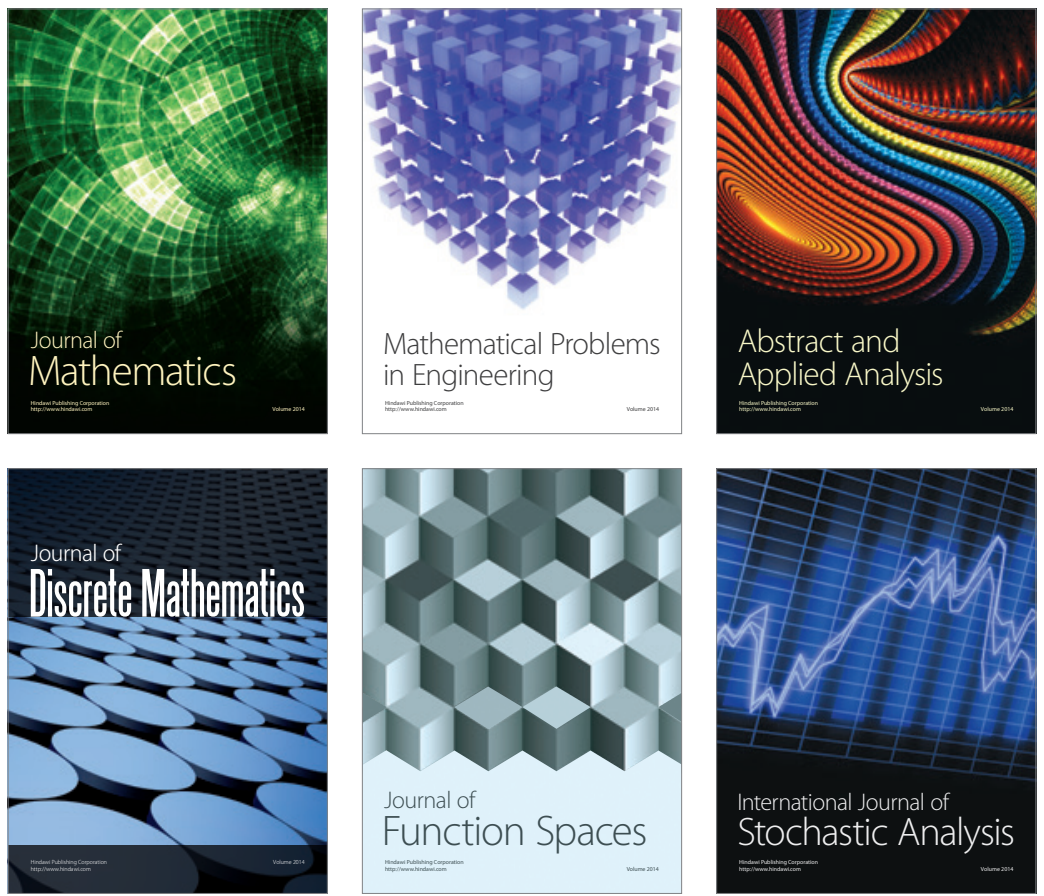

Journal of

Function Spaces

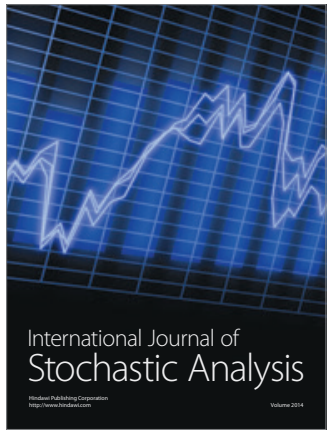

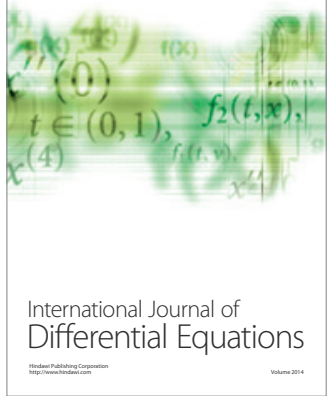
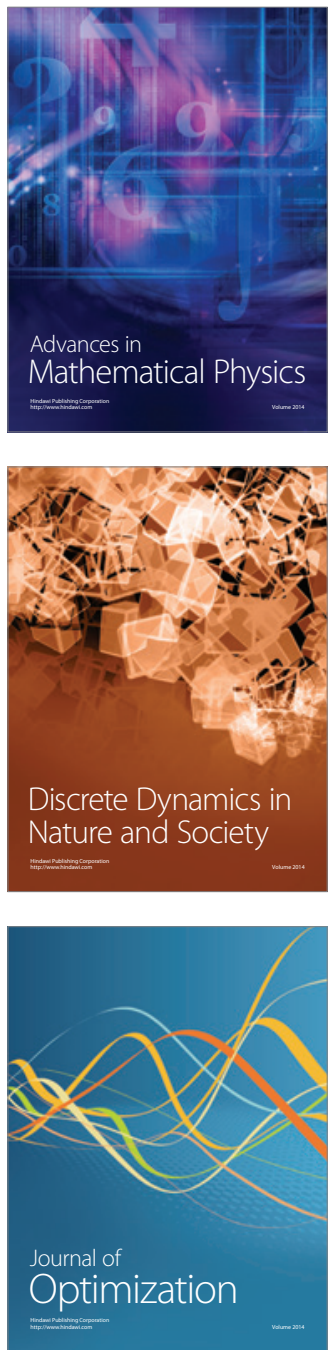